\title{
A reconstruction method based on topological derivatives for an inverse problem modeled by the Helmholtz equation
}

\author{
Lucas dos Santos Fernandez ${ }^{1}$, Antonio André Novotny ${ }^{2}$ \\ Laboratório Nacional de Computação Científica, LNCC/MCTIC, Petrópolis, RJ, Brasil \\ Ravi Prakash ${ }^{3}$ \\ Facultad de Ciencias Físicas y Matemáticas, Universidad de Concepción, Concepción, Chile
}

\begin{abstract}
This paper deals with an inverse potential problem whose forward problem is governed by the Helmholtz equation. The inverse problem consists in the reconstruction of a set of anomalies embedded into a fluid medium with the help of partial measurements of the associated potential. We rewrite the inverse problem as a topology optimization problem which allows us to solve it by using the concept of topological derivatives. The resulting algorithm is able to reconstruct the anomalies in one step and is independent of any initial guess. A numerical example is presented to show the effectiveness of our reconstruction method.
\end{abstract}

Keywords. Inverse potential problem, Helmholtz equation, higher-order topological derivatives, topology optimization, reconstruction method.

\section{Introduction}

In this paper, we deal with an inverse potential problem in $\mathbb{R}^{2}$ whose corresponding forward problem is governed by the Helmholtz equation. The inverse problem under consideration is about the reconstruction of a set of anomalies embedded in a geometrical domain with the help of partial measurements of the associated potential taken on its boundary. More precisely, let $\Omega \subset \mathbb{R}^{2}$ be an open and bounded domain with smooth boundary $\partial \Omega$ where the measurements of a scalar field of interest are taken. We assume that there may be an unknown number $\left(N^{*} \in \mathbb{Z}^{+}\right)$of isolated anomalies $\omega_{i}^{*}$ within the domain $\Omega$, i.e., there is a set $\omega^{*}=\cup_{i=1}^{N^{*}} \omega_{i}^{*}$, with open connected components $\omega_{i}^{*}$ which satisfy $\overline{\omega_{i}^{*}} \cap \overline{\omega_{j}^{*}}=\emptyset$ for $i \neq j$ and $\overline{\omega_{i}^{*}} \cap \partial \Omega=\emptyset$ for each $i, j \in\left\{1, \ldots, N^{*}\right\}$.

We consider the domain $\Omega$ as a bounded region representing a fluid medium which contains a different fluid substance within a subdomain $\omega^{*}$. For a given Robin data $g_{R}$ imposed on $\partial \Omega$, the resulting substance concentration (potential) $z$ in $\Omega$ is observed on $\partial \Omega$. In this set up, the inverse problem consists in finding $k_{\omega^{*}}$ such that the substance

\footnotetext{
${ }^{1}$ lucasf@lncc.br

${ }^{2}$ novotny@lncc.br

${ }^{3}$ rprakash@udec.cl
} 
concentration $z$ satisfies the following boundary value problem

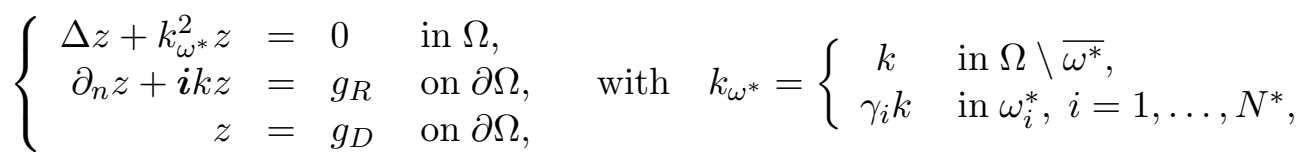

where $g_{R}$ and $g_{D}$ are the boundary excitation and boundary measurement, respectively; and $\boldsymbol{i}$ is the imaginary number. In addition, $k, \gamma_{i} \in \mathbb{R}^{+}$, where $\gamma_{i}$ is the contrast with respect to the material property of the background $k$. Now, for an initial guess $k_{\omega}$ of $k_{\omega^{*}}$, we consider the substance concentration field $u$ to be the solution to the boundary value problem

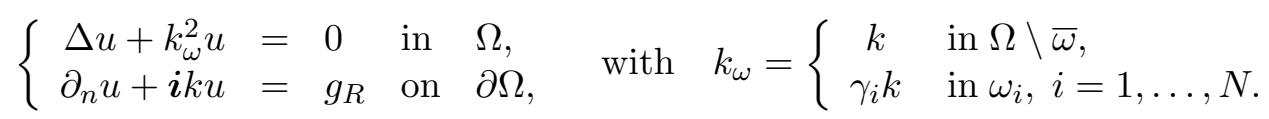

Since we want our guess $k_{\omega}$ to be close to the unknown $k_{\omega^{*}}$, it is natural to wish that the scalar fields $u$ and $z$ have the same measurement on $\partial \Omega$. Keeping this objective in mind, we rewrite the inverse problem in the form of the following topology optimization problem given by

$$
\underset{\omega \subset \Omega}{\operatorname{Minimize}} \mathcal{J}_{\omega}\left(u^{1}, \ldots, u^{M}\right)=\sum_{m=1}^{M} \int_{\partial \Omega}\left(u^{m}-z^{m}\right) \overline{\left(u^{m}-z^{m}\right)}
$$

where $M \in \mathbb{Z}^{+}$is the number of observations, $z^{m}$ and $u^{m}$ are the solutions of the boundary value problems (1) and (2), respectively, corresponding to the Robin data $g_{R}^{m}$ with $m=$ $1, \ldots, M$. Moreover, $\overline{(\cdot)}$ represents the complex conjugate of $(\cdot)$. Notice that, the minimizer of the topology optimization problem (3) produces the best approximation to $\omega^{*}$, solution of the inverse problem (1), in an appropriate sense. In particular, problem (3) is minimized with respect to a set of ball-shaped anomalies by using the concept of topological derivatives [4]. It means that the shape functional $\mathcal{J}_{\omega}\left(u^{1}, \ldots, u^{M}\right)$ is expanded asymptotically and then truncated up to the desired order term. The resulting expression is trivially minimized with respect to the parameters under consideration, leading us to a noniterative second-order reconstruction algorithm.

The remaining part of this paper is organized as follows. Since the proposed inverse problem is to be solved by using the concept of topological derivatives, we present in Section 2 the shape functionals corresponding to the unperturbed and perturbed domains as well as the ansätz for the scalar field associated to the topologically perturbed domain. In Section 3, the asymptotic expansion of the shape functional is presented. Based on such asymptotic expansion of the shape functional, a noniterative reconstruction algorithm is devised in Section 4 and a numerical example is presented in Section 5. Concluding remarks are discussed in Section 6.

\section{Topology optimization setting}

The inverse problem (1) has been written in the form of the topology optimization problem (3). It is well known that a quite general approach for dealing with such class 
of problems is based on the concept of topological derivative, which consists in expanding the shape functional $\mathcal{J}_{\omega}\left(u^{1}, \ldots, u^{M}\right)$ with respect to the parameters depend upon a set of small inclusions. The reader may refer to the book of Novotny \& Sokołowski [1] to be familiar with the notion of topological derivatives. Since the topological derivative does not depend on the initial guess of the unknown topology $\omega^{*}$, we start with the unperturbed domain by setting $\omega=\emptyset$. More precisely, we consider

$$
\mathcal{J}_{0}\left(u_{0}^{1}, \ldots, u_{0}^{M}\right)=\sum_{m=1}^{M} \int_{\partial \Omega}\left(u_{0}^{m}-z^{m}\right) \overline{\left(u_{0}^{m}-z^{m}\right)},
$$

where $u_{0}^{m}$ be the solution of the unperturbed boundary value problem

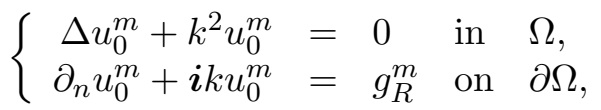

Here, we are considering the topology optimization problem (3) for ball-shaped perturbations and hence we define the topologically perturbed counter-part of (5) by introducing $N \in \mathbb{Z}^{+}$number of small circular inclusions $B_{\varepsilon_{i}}\left(x_{i}\right)$ with center at $x_{i} \in \Omega$ and radius $\varepsilon_{i}$ for $i=1, \ldots, N$. The set of inclusions is denoted as $B_{\varepsilon}(\xi)=\cup_{i=1}^{N} B_{\varepsilon_{i}}\left(x_{i}\right)$, where $\xi=\left(x_{1}, \ldots, x_{N}\right)$ and $\varepsilon=\left(\varepsilon_{1}, \ldots, \varepsilon_{N}\right)$. Moreover, we assume that $\overline{B_{\varepsilon}} \cap \partial \Omega=\emptyset$ and $\overline{B_{\varepsilon_{i}}\left(x_{i}\right)} \cap \overline{B_{\varepsilon_{j}}\left(x_{j}\right)}=\emptyset$ for each $i \neq j$ and $i, j \in\{1, \ldots, N\}$. The shape functional associated with the topologically perturbed domain is written as

$$
\mathcal{J}_{\varepsilon}\left(u_{\varepsilon}^{1}, \ldots, u_{\varepsilon}^{M}\right)=\sum_{m=1}^{M} \int_{\partial \Omega}\left(u_{\varepsilon}^{m}-z^{m}\right) \overline{\left(u_{\varepsilon}^{m}-z^{m}\right)}
$$

with $u_{\varepsilon}^{m}$ be the solution of the perturbed boundary value problem

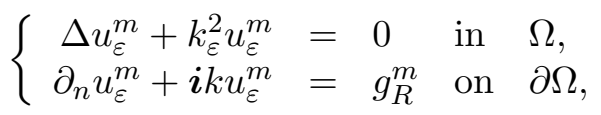

where the parameter $k_{\varepsilon}$ is defined as

$$
k_{\varepsilon}=\left\{\begin{array}{cl}
k & \text { in } \Omega \backslash \cup_{i=1}^{N} \overline{B_{\varepsilon_{i}}\left(x_{i}\right)}, \\
\gamma_{i} k & \text { in } B_{\varepsilon_{i}}\left(x_{i}\right)
\end{array}\right.
$$

In order to obtain the topological derivatives of the shape functional $\mathcal{J}_{\varepsilon}$ at $u_{\varepsilon}$, we start by simplifying the difference between the perturbed shape functional $\mathcal{J}_{\varepsilon}\left(u_{\varepsilon}^{1}, \ldots, u_{\varepsilon}^{M}\right)$ and its unperturbed counter-part $\mathcal{J}_{0}\left(u_{0}^{1}, \ldots, u_{0}^{M}\right)$ defined in (6) and (4), respectively, as follows

$$
\mathcal{J}_{\varepsilon}\left(u_{\varepsilon}\right)-\mathcal{J}_{0}\left(u_{0}\right)=\sum_{m=1}^{M} \int_{\partial \Omega}\left[2 \Re\left\{\left(u_{\varepsilon}^{m}-u_{0}^{m}\right) \overline{\left(u_{0}^{m}-z^{m}\right)}\right\}+\left(u_{\varepsilon}^{m}-u_{0}^{m}\right) \overline{\left(u_{\varepsilon}^{m}-u_{0}^{m}\right)}\right],
$$

where $u_{\varepsilon}=\left(u_{\varepsilon}^{1}, \ldots, u_{\varepsilon}^{M}\right), u_{0}=\left(u_{0}^{1}, \ldots, u_{0}^{M}\right)$ and $\mathfrak{R}\{\cdot\}$ denotes the real part of $\{\cdot\}$. Moreover, we first introduce the quantity $\beta_{i}=\gamma_{i}^{2}-1$ and the vector $\alpha \in \mathbb{R}^{N}$ where each entry $\alpha_{i}$ denotes the Lebesgue measure (volume) of the two-dimensional ball $B_{\varepsilon_{i}}\left(x_{i}\right)$, i.e.,

$$
\alpha=\left(\alpha_{1}, \ldots, \alpha_{N}\right) \quad \text { with } \quad \alpha_{i}=\left|B_{\varepsilon_{i}}\left(x_{i}\right)\right|=\pi \varepsilon_{i}^{2}, \quad \text { for } \quad i=1, \ldots, N .
$$


Now, we propose the following ansätz for the expansion of $u_{\varepsilon}^{m}$ with respect to the parameters corresponding to the small circular inclusions in the form

$$
u_{\varepsilon}^{m}(x)=u_{0}^{m}(x)+k^{2} \sum_{i=1}^{N} \alpha_{i} \beta_{i} h_{i}^{\varepsilon, m}(x)+k^{4} \sum_{i=1}^{N} \sum_{j=1}^{N} \alpha_{i} \alpha_{j} \beta_{i} \beta_{j} h_{i j}^{\varepsilon, m}(x)+\tilde{u}_{\varepsilon}^{m}(x),
$$

where, for each $i, j=1, \ldots, N$ and $m=1, \ldots, M$, the functions $h_{i}^{\varepsilon, m}, h_{i j}^{\varepsilon, m}$ and $\tilde{u}_{\varepsilon}^{m}$ are the solutions of

$$
\begin{aligned}
& \left\{\begin{aligned}
\Delta h_{i}^{\varepsilon, m}+k^{2} h_{i}^{\varepsilon, m} & =-\alpha_{i}^{-1} u_{0}^{m} \chi_{B_{\varepsilon_{i}}\left(x_{i}\right)} & & \text { in } \Omega, \\
\partial_{n} h_{i}^{\varepsilon, m}+i k h_{i}^{\varepsilon, m} & =0 & & \text { on } \partial \Omega,
\end{aligned}\right. \\
& \left\{\begin{aligned}
\Delta h_{i j}^{\varepsilon, m}+k^{2} h_{i j}^{\varepsilon, m} & =-\alpha_{i}^{-1} h_{j}^{\varepsilon, m} \chi_{B_{\varepsilon_{i}}\left(x_{i}\right)} & \text { in } \Omega, \\
\partial_{n} h_{i j}^{\varepsilon, m}+i k h_{i j}^{\varepsilon, m} & =0 & \text { on } \partial \Omega,
\end{aligned}\right.
\end{aligned}
$$

and

$$
\left\{\begin{aligned}
\Delta \tilde{u}_{\varepsilon}^{m}+k_{\varepsilon}^{2} \tilde{u}_{\varepsilon}^{m} & =\Phi_{\varepsilon}^{m} & & \text { in } \Omega \\
\partial_{n} \tilde{u}_{\varepsilon}^{m}+i k \tilde{u}_{\varepsilon}^{m} & =0 & & \text { on } \partial \Omega
\end{aligned}\right.
$$

respectively. In problem (14), we have $\Phi_{\varepsilon}^{m}=-k^{6} \sum_{i, j, l=1}^{N} \alpha_{j} \alpha_{l} \beta_{i} \beta_{j} \beta_{l} h_{j l}^{\varepsilon, m} \chi_{B_{\varepsilon_{i}}\left(x_{i}\right)}$. In order to simplify further analysis, we write $h_{i}^{\varepsilon, m}$ as a sum of three functions $p_{i}^{\varepsilon}, q_{i}^{\varepsilon}$ and $\tilde{h}_{i}^{\varepsilon, m}$ in the form $h_{i}^{\varepsilon, m}=u_{0}^{m}\left(x_{i}\right) h_{i}^{\varepsilon}+\tilde{h}_{i}^{\varepsilon, m}$ with $h_{i}^{\varepsilon}=p_{i}^{\varepsilon}+q_{i}^{\varepsilon}$. The function $p_{i}^{\varepsilon}$ is solution of

$$
\left\{\begin{aligned}
\Delta p_{i}^{\varepsilon}+k^{2} p_{i}^{\varepsilon} & =-\alpha_{i}^{-1} \chi_{B_{\varepsilon_{i}}\left(x_{i}\right)} & & \text { in } B_{R}\left(x_{i}\right), \\
p_{i}^{\varepsilon} & =\lambda_{3}^{\varepsilon_{i}} Y_{0}(k R) & & \text { on } \partial B_{R}\left(x_{i}\right),
\end{aligned}\right.
$$

with $B_{\varepsilon_{i}}\left(x_{i}\right) \subset \Omega \subset B_{R}\left(x_{i}\right), x_{i} \in \Omega, 0<\varepsilon_{i} \ll R$. Moreover, $\lambda_{3}^{\varepsilon_{i}}$ denotes a constant depending on $\varepsilon_{i}$ to be presented next. Problem (15) can be solved analytically and its solution is written in terms of Bessel functions, namely,

$$
p_{i}^{\varepsilon}(x)= \begin{cases}\lambda_{1}^{\varepsilon_{i}}+\lambda_{2}^{\varepsilon_{i}} J_{0}\left(k\left\|x-x_{i}\right\|\right) & \text { in } B_{\varepsilon_{i}}\left(x_{i}\right), \\ \lambda_{3}^{\varepsilon_{i}} Y_{0}\left(k\left\|x-x_{i}\right\|\right) & \text { in } B_{R}\left(x_{i}\right) \backslash \overline{B_{\varepsilon_{i}}\left(x_{i}\right)},\end{cases}
$$

with the following constants depending on the small parameter $\varepsilon_{i}$,

$$
\lambda_{1}^{\varepsilon_{i}}=-\frac{1}{k^{2} \pi \varepsilon_{i}^{2}}, \quad \lambda_{2}^{\varepsilon_{i}}=\frac{1}{k^{2} \pi \varepsilon_{i}^{2}}+\lambda-\frac{1}{2 \pi} \ln \varepsilon_{i}+\tilde{\lambda}_{2}^{\varepsilon_{i}} \quad \text { and } \quad \lambda_{3}^{\varepsilon_{i}}=-\frac{1}{4}+\tilde{\lambda}_{3}^{\varepsilon_{i}} .
$$

In (17), $\lambda=(2 \ln 2-2 \ln k-2 \zeta+1) /(4 \pi), \zeta$ is the Euler constant, $\tilde{\lambda}_{2}^{\varepsilon_{i}}=O\left(\varepsilon_{i}^{2}\right)$ and $\tilde{\lambda}_{3}^{\varepsilon_{i}}=O\left(\varepsilon_{i}^{2}\right)$. Taking into account the solution $p_{i}^{\varepsilon}$ of the problem (15), we write $q_{i}^{\varepsilon}=\lambda_{3}^{\varepsilon_{i}} q_{i}$, where $q_{i}$ is the solution to the homogeneous boundary value problem

$$
\left\{\begin{aligned}
\Delta q_{i}+k^{2} q_{i} & =0 & & \text { in } \Omega, \\
\partial_{n} q_{i}+\boldsymbol{i} k q_{i} & =-\left(\partial_{n} Y_{0}\left(k\left\|x-x_{i}\right\|\right)+\boldsymbol{i} k Y_{0}\left(k\left\|x-x_{i}\right\|\right)\right) & & \text { on } \partial \Omega,
\end{aligned}\right.
$$

and $\tilde{h}_{i}^{\varepsilon, m}$ solves the boundary value problem

$$
\left\{\begin{aligned}
\Delta \tilde{h}_{i}^{\varepsilon, m}+k^{2} \tilde{h}_{i}^{\varepsilon, m} & =-\alpha_{i}^{-1}\left(u_{0}^{m}-u_{0}^{m}\left(x_{i}\right)\right) \chi_{B_{\varepsilon_{i}}\left(x_{i}\right)} & & \text { in } \Omega, \\
\partial_{n} \tilde{h}_{i}^{\varepsilon, m}+\boldsymbol{i} k \tilde{h}_{i}^{\varepsilon, m} & =0 & & \text { on } \partial \Omega .
\end{aligned}\right.
$$

Moreover, we also introduce an adjoint state $v^{m}$ as the solution of the following auxiliary boundary value problem

$$
\left\{\begin{aligned}
\Delta v^{m}+k^{2} v^{m} & =0 & & \text { in } \Omega, \\
\partial_{n} v^{m}-i k v^{m} & =-\left(u_{0}^{m}-z^{m}\right) & & \text { on } \partial \Omega .
\end{aligned}\right.
$$




\section{Topological asymptotic expansion}

Now, we have all the elements to evaluate the difference (9) explicitly. We proceed accordingly to the following sequence of steps: (i) the scalar field $u_{\varepsilon}^{m}$ in (9) is replaced by the expression given by the ansätz introduced in (11); (ii) the weak formulations of the problems (12), (13) and (20) are used in order to rewrite the integrals defined over the boundary $\partial \Omega$ by integrals defined over the ball $B_{\varepsilon_{i}}\left(x_{i}\right)$; and (iii) the decomposition $h_{i}^{\varepsilon, m}=u_{0}^{m}\left(x_{i}\right)\left(p_{i}^{\varepsilon}+\lambda_{3}^{\varepsilon_{i}} q_{i}\right)+\tilde{h}_{i}^{\varepsilon, m}$ is then used taking into account the analytical solution for $p_{i}^{\varepsilon}$, given by (16)-(17), together with Taylor's expansions for the functions $u_{0}^{m}, v^{m}$ and $p_{j}^{\varepsilon}$ around the point $x_{i}$ (the center of the ball $B_{\varepsilon_{i}}$ ). As a result, we obtain the asymptotic expansion of the shape functional $\mathcal{J}_{\omega}\left(u^{1}, \ldots, u^{M}\right)$ in its matrix form given by

$$
\psi\left(\chi_{\varepsilon}(\xi)\right)=\psi(\chi)-\alpha \cdot d(\xi)+G(\xi) \alpha \cdot \operatorname{diag}(\alpha \otimes \ln \alpha)+\frac{1}{2} H(\xi) \alpha \cdot \alpha+o\left(|\alpha|^{2}\right),
$$

where $\psi\left(\chi_{\varepsilon}(\xi)\right):=\mathcal{J}_{\varepsilon}\left(u_{\varepsilon}\right)$ and $\psi(\chi):=\mathcal{J}_{0}\left(u_{0}\right)$; the entries of the vector $d \in \mathbb{R}^{N}$ and the matrices $G, H \in \mathbb{R}^{N \times N}$ are defined as

$$
\begin{gathered}
d_{i}:=2 k^{2} \beta_{i} \sum_{m=1}^{M} \mathfrak{R}\left\{u_{0}^{m}\left(x_{i}\right) \overline{v^{m}}\left(x_{i}\right)\right\}, \\
G_{i i}:=\frac{1}{2 \pi} k^{4} \beta_{i}^{2} \sum_{m=1}^{M} \mathfrak{R}\left\{u_{0}^{m}\left(x_{i}\right) \overline{v^{m}}\left(x_{i}\right)\right\}, \quad G_{i j}=0, \quad \text { if } \quad i \neq j
\end{gathered}
$$

and

$$
\begin{aligned}
H_{i i}:= & \frac{1}{\pi} k^{4} \beta_{i} \sum_{m=1}^{M} \mathfrak{R}\left\{u_{0}^{m}\left(x_{i}\right) \overline{v^{m}}\left(x_{i}\right)\right\}-\frac{1}{\pi} k^{2} \beta_{i} \sum_{m=1}^{M} \mathfrak{R}\left\{\nabla u_{0}^{m}\left(x_{i}\right) \cdot \nabla \overline{v^{m}}\left(x_{i}\right)\right\} \\
& -\frac{1}{2 \pi} \sigma k^{4} \beta_{i}^{2} \sum_{m=1}^{M} \mathfrak{R}\left\{u_{0}^{m}\left(x_{i}\right) \overline{v^{m}}\left(x_{i}\right)\right\}+k^{4} \beta_{i}^{2} \sum_{m=1}^{M} \mathfrak{R}\left\{u_{0}^{m}\left(x_{i}\right) \overline{v^{m}}\left(x_{i}\right) q_{i}\left(x_{i}\right)\right\} \\
& +\frac{1}{8} k^{4} \beta_{i}^{2} \sum_{m=1}^{M} u_{0}^{m}\left(x_{i}\right) \overline{u_{0}^{m}}\left(x_{i}\right) \mathcal{I}_{i i}, \\
H_{i j}:= & k^{4} \beta_{i} \beta_{j} \sum_{m=1}^{M} \mathfrak{R}\left\{u_{0}^{m}\left(x_{j}\right) \overline{v^{m}}\left(x_{i}\right) \mathcal{Y}_{i j}\right\}+k^{4} \beta_{i} \beta_{j} \sum_{m=1}^{M} \mathfrak{R}\left\{u_{0}^{m}\left(x_{j}\right) \overline{v^{m}}\left(x_{i}\right) q_{j}\left(x_{i}\right)\right\} \\
& +\frac{1}{8} k^{4} \beta_{i} \beta_{j} \sum_{m=1}^{M} \mathfrak{R}\left\{u_{0}^{m}\left(x_{i}\right) \overline{u_{0}^{m}}\left(x_{j}\right) \mathcal{I}_{i j}\right\},
\end{aligned}
$$

if $i \neq j$; respectively, for $i, j=1, \ldots, N$. In addition, the entries (24)-(25) are written in terms of the constant $\sigma=1-4 \zeta+\ln \left(16 \pi^{2} / k^{4}\right)$, the number $\mathcal{Y}_{i j}=Y_{0}\left(k\left\|x_{i}-x_{j}\right\|\right)$ and the integral

$$
\mathcal{I}_{i j}=\int_{\partial \Omega}\left[Y_{0}\left(k\left\|x-x_{i}\right\|\right)+q_{i}(x)\right]\left[\overline{Y_{0}}\left(k\left\|x-x_{j}\right\|\right)+\overline{q_{j}}(x)\right],
$$

where $Y_{0}$ denotes the Bessel function of the second kind and zero order. 


\section{Reconstruction algorithm}

The expression on the right-hand side of (21) depends on the number of anomalies $N$, their sizes $\alpha$ and locations $\xi$. Thus, by desregarding the terms of order $o\left(|\alpha|^{2}\right)$ from (21), we define $\delta J(\alpha, \xi, N):=-\alpha \cdot d(\xi)+G(\xi) \alpha \cdot \operatorname{diag}(\alpha \otimes \ln \alpha)+1 / 2 H(\xi) \alpha \cdot \alpha$. The derivative of the function $\delta J(\alpha, \xi, N)$ with respect to the variable $\alpha$ yields the first-order optimality condition, namely, $\left\langle D_{\alpha} \delta J, \beta\right\rangle=0, \forall \beta \in \mathbb{R}^{N}$, which leads to the non-linear system of the form

$$
(H(\xi)+G(\xi)) \alpha+2 G(\xi) \operatorname{diag}(\alpha \otimes \ln \alpha)=d(\xi)
$$

with the entries of the vector $d \in \mathbb{R}^{N}$ and the matrices $G, H \in \mathbb{R}^{N \times N}$ defined in (22), (23) and (24)-(25), respectively.

The quantity $\alpha$ solution of (27) becomes a function of the locations $\xi$, namely $\alpha=\alpha(\xi)$, and its value is obtained by using the Newton's method. Now, let us replace the solution of (27) into $\delta J(\alpha, \xi, N)$ to obtain $\delta J(\alpha(\xi), \xi, N)=-\frac{1}{2}(d(\xi)+G(\xi) \alpha(\xi)) \cdot \alpha(\xi)$. Therefore, the pair of vectors $\left(\xi^{\star}, \alpha^{\star}\right)$ which minimizes $\delta J(\alpha, \xi, N)$ is given by $\xi^{\star}=$ $\operatorname{argmin}_{\xi \in X} \delta J(\alpha(\xi), \xi, N)$ and $\alpha^{\star}:=\alpha\left(\xi^{\star}\right)$, where $X$ is the set of admissible locations for anomalies. In other words, the minimizer of $\delta J(\alpha, \xi, N)$ is a set of ball-shaped disjoint inclusions which is completely characterized by the pair $\left(\xi^{\star}, \alpha^{\star}\right)$. In short, for a given number of anomalies $N$ we want to reconstruct, our method is able to find in one step their sizes $\alpha^{\star}$ and their locations $\xi^{\star}$. For more sophisticated approaches based on metaheuristic and multi-grid methods, we refer to [3], where the algorithm proposed in this section can be found in pseudo-code format. For more applications of this algorithm, see [2] (Chapter 10), for instance.

\section{$5 \quad$ Numerical example}

A numerical example is presented here to demonstrate the effectiveness of the method proposed in the earlier sections of this paper.

The reference domain is taken as a unitary disk centered at the origin, namely $\Omega:=$ $B_{1}(0)$, which is discretized using a three-node finite element scheme. The boundary of the geometrical domain $\partial \Omega$ is excited by imposing different Robin data whose mathematical expression is given by $g_{R}^{m}=\exp \left(-\boldsymbol{i} k\left(x \cos \theta_{m}+y \sin \theta_{m}\right)\right)$ with $\theta_{m}=(m-1) \pi / M$, where $m=1, \ldots, M$. The associated potential $g_{D}^{m}$, for $m=1, \ldots, M$, is measured on the whole boundary $\partial \Omega$. For a number $M$ of measurements, the objective is to reconstruct a number $N^{*}$ of anomalies with contrast $\gamma_{i}=\gamma, \gamma \in \mathbb{R}^{+}$, for $i=1, \ldots, N^{*}$, from the help of measurements of the potential $g_{D}^{m}$ taken in $\partial \Omega$. The auxiliary boundary value problems are solved using the Finite Element Method. However, due to the high complexity of the reconstruction algorithm, the sub-mesh $X$ is defined over the finite element mesh where the combinatorial search is performed in order to find the optimal size $\alpha^{\star}$ and the appropriate center $\xi^{\star}$ of the geometrical domain $\omega^{*}$.

The example: Two circular regions with centers located at $x_{1}^{*}=(-0.4,0.5), x_{2}^{*}=$ $(0.2,-0.5)$ whose radii are given by $\varepsilon_{1}^{*}=0.03$ and $\varepsilon_{2}^{*}=0.05$, respectively, are considered as the target anomalies. See Figure 1(a). The geometrical domain $\Omega$ is disctretized into 
198912 elements and 99753 nodes. The combinatorial search was conducted on a sub-mesh of 371 nodes distributed within the domain $\Omega$. In the current setting, the reconstruction is performed by taking into account only one measurement obtained from the Robin data $g_{R}^{1}\left(\theta_{1}=0\right)$. By comparing Figures $1(\mathrm{a})$ and $1(\mathrm{~b})$, one can observe that we failed in the reconstruction of both anomalies. In fact, although the obtained sizes are close to the true ones, the centers do not match. This happens because the lack of information. Therefore, we improve the number of measurements by considering three Robin data simultaneously, namely, $g_{R}^{1}, g_{R}^{2}\left(\theta_{2}=\pi / 3\right)$ and $g_{R}^{3}\left(\theta_{3}=2 \pi / 3\right)$. In this case, anomalies are accurately reconstructed, as we can observe by comparing Figures 1(a) and 1(c).

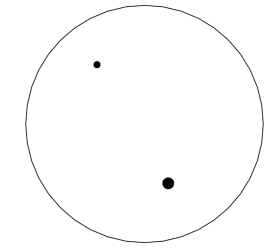

(a) Target

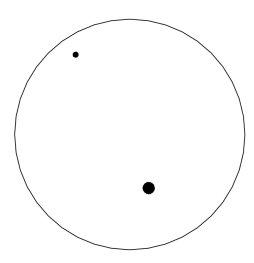

(b) Result $M=1$

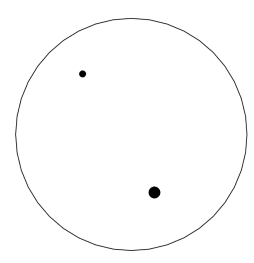

(c) Result $M=3$

Figure 1: Example: Target and results.

\section{Conclusions}

In this paper, a noniterative reconstruct method for an inverse potential problem modeled by the Helmholtz equation is proposed. The method is based on the topological derivatives of the shape functional associated with the inverse problem. The algorithm devised is able to reconstruct the embedded anomalies in one step and is independent of any initial guess. In addition, we conclude from the above example that more accurate results may require more than one observation depending on the number of embedded anomalies to be reconstructed.

\section{References}

[1] A. A. Novotny and J. Sokołowski. Topological derivatives in shape optimization, Interactions of Mechanics and Mathematics. Springer-Verlag, Berlin, Heidelberg, 2013.

[2] A. A. Novotny, J. Sokołowski and A. Żochowski. Applications of the topological derivative method, Studies in Systems, Decision and Control. Springer Nature, Switzerland, 2019 .

[3] T. J. Machado, J. S. Angelo and A. A. Novotny. A new one-shot pointwise source reconstruction method, Mathematical Methods in Applied Sciences, 40:1367-1381, 2017.

[4] J. Sokołowski and A. Żochowski. On the topological derivative in shape optimization, SIAM Journal on Control and Optimization, 37(4):1251-1272, 1999. 